\title{
Immune Thrombocytopenic Purpura Secondary to Histoplasmosis
}

\author{
Anupam Gupta ${ }^{\mathrm{a}}$, Ivan Kamikovski ${ }^{\mathrm{a}}$, Armin Kamyab ${ }^{\mathrm{a}}$, Michael Jacobs ${ }^{\mathrm{a}}$, b
}

\begin{abstract}
Immune thrombocytopenic purpura (ITP) is a relatively common acquired bleeding disorder characterized by autoimmune destruction of platelets. In adults, the annual incidence is around 1.6/100,000, common in middle aged females. In patients with ITP, physical examination is usually normal aside from bleeding manifestations, such as petechiae, ecchymoses, and purpura. The three key diagnostic criteria for ITP are isolated thrombocytopenia with otherwise normal peripheral complete blood count and smear, an absence of hepatosplenomegaly and lymphadenopathy on physical examination, and platelet response to classic ITP therapy. Review of the literature has shown case reports of disseminated histoplasmosis presenting with thrombocytopenia. Our patient comes from a geographic location where histoplasmosis is endemic. Most cases of histoplasmosis have mild symptoms and usually escape diagnosis. The microconidia formed in the mold phase of Histoplasma capsulatum are easily aerosolized, inhaled into the lungs, and then phagocytized by alveolar macrophages. Inside the macrophage, the organism converts to the yeast phase, survives within the macrophage for the first few weeks, and disseminates widely throughout the reticuloendothelial system. If cellmediated immunity is deficient because of immunosuppression due to corticosteroids, transplanted organs, the organisms remain viable within macrophages and cause progressive infection. Most patients have either no symptoms or mild pulmonary complaints that are not severe enough to seek medical care. Although disseminated histoplasmosis has been reported to be associated with thrombocytopenia and anemia, most of these patients are immunocompromised. We report a case of ITP secondary to histoplasmosis in an immunocompetent patient.
\end{abstract}

Keywords: ITP; Histoplasmosis; Methenamine silver stain

Manuscript accepted for publication July 09, 2015

aDepartment of Surgery, Providence Hospital, St. John Providence Health, Southfield, MI, USA

${ }^{b}$ Corresponding Author: Michael J. Jacobs, Department of Surgery, Providence Hospital and Medical Centers, 16001 W. Nine Mile Road, Southfield, MI 48075, USA. Email: mjjacobsmd@gmail.com

doi: http://dx.doi.org/10.14740/jmc2230w

\section{Introduction}

Idiopathic thrombocytopenic purpura (ITP) is an antibodymediated destruction of platelets by the reticuloendothelial system. The triggering etiology in most cases is unknown, but it has been reported to be associated with certain infections such as hepatitis $\mathrm{C}$ virus (HCV), human immunodeficiency virus (HIV), Ebstein-Barr virus (EBV) and Helicobacter pylori. Histoplasmosis is the most common of all North American fungal infections. The lymphogenous reaction to Histoplasma can cause mediastinal lymph node enlargement, bronchiectasis, constrictive pericarditis, and rarely, idiopathic thrombocytopenia. We report a case of ITP secondary to histoplasmosis in an immunocompetent patient.

\section{Case Report}

A 63-year-old Caucasian female presented to the emergency department with a 1-week history of non-bloody diarrhea, lightheadedness, and nausea. The patient's past medical history was significant for ITP diagnosed in 1990 with a relapse in 2011 during which time she was treated with aggressive medical management. During this most recent admission, she was found to have a platelet count of $6,000 / \mu \mathrm{L}$ and therefore diagnosed as having another ITP relapse.

The patient was admitted and started on intravenous immunoglobulin and steroids. Her platelet count gradually improved and the patient was evaluated for a splenectomy as definitive treatment of her ITP. Two weeks after discharge, the patient underwent a splenectomy.

The patient tolerated the procedure well, without any intraoperative complications. Pathologic evaluation of the resected spleen revealed methenamine silver stain-positive structures consistent with Histoplasma species (Fig. 1). The patient was therefore diagnosed as having ITP secondary to histoplasmosis, a rare but known risk factor for the development of ITP.

\section{Discussion}

In 1951, William Harrington published and first described patients with ITP [1]. Fifty years later, many aspects of the pathogenesis of ITP remain unanswered, and the management 


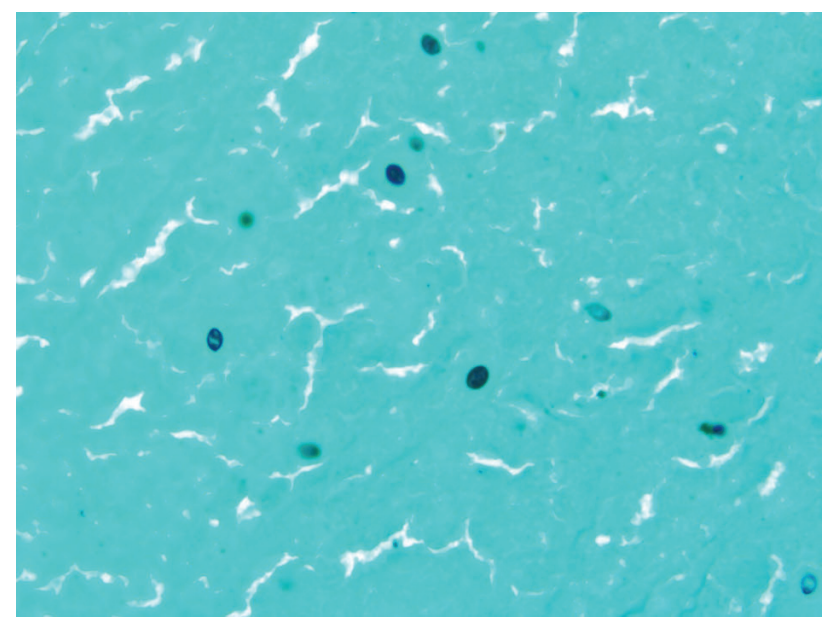

Figure 1. Methenamine silver stain-positive structures consistent with Histoplasma species given the morphology and location $(\times 1,000)$.

remains controversial [1].

ITP is a relatively common acquired bleeding disorder characterized by autoimmune destruction of platelets. In adults, the annual incidence is around $1.6 / 100,000$, common in middle aged females [1]. In patients with ITP, physical examination is usually normal aside from bleeding manifestations, such as petechiae, ecchymoses, and purpura [1]. The three key diagnostic criteria for ITP are isolated thrombocytopenia with otherwise normal peripheral complete blood count and smear, an absence of hepatosplenomegaly and lymphadenopathy on physical examination, and platelet response to classic ITP therapy [1].

ITP has been noted to be associated with HIV, H. pylori, and HCV as possible etiological agents in some cases [1]. Histoplasma is frequently seen in certain geographic location associated with decaying bat guano or bird droppings [2]. In the United States, it is commonly seen in the states bordering the Ohio River valley and the lower Mississippi River [2]. Histoplasmosis commonly involves the lungs and mimics sarcoido- sis. Disseminated histoplasmosis is usually seen in association with immunosuppressed individuals $[3,4]$.

Review of the literature has shown case reports of disseminated histoplasmosis presenting with thrombocytopenia [4]. Our patient comes from a geographic location where histoplasmosis is endemic. Most cases of histoplasmosis have mild symptoms and usually escape diagnosis [4].

The microconidia formed in the mold phase of Histoplasma capsulatum are easily aerosolized, inhaled into the lungs, and then phagocytized by alveolar macrophages. Inside the macrophage, the organism converts to the yeast phase, survives within the macrophage for the first few weeks, and disseminates widely throughout the reticuloendothelial system [3]. If cell-mediated immunity is deficient because of immunosuppression due to corticosteroids, transplanted organs, the organisms remain viable within macrophages and cause progressive infection [3]. Most patients have either no symptoms or mild pulmonary complaints that are not severe enough to seek medical care [3].

Although disseminated histoplasmosis has been reported to be associated with thrombocytopenia and anemia, most of these patients are immunocompromised. We report a case of ITP secondary to histoplasmosis in an immunocompetent patient.

\section{References}

1. Psaila B, Bussel JB. Immune thrombocytopenic purpura. Hematol Oncol Clin North Am. 2007;21(4):743-759, vii.

2. Di Paola JA, Buchanan GR. Immune thrombocytopenic purpura. Pediatr Clin North Am. 2002;49(5):911-928.

3. Wheat LJ, Kauffman CA. Histoplasmosis. Infect Dis Clin North Am. 2003;17(1):1-19, vii.

4. Hood AB, Inglis FG, Lowenstein L, Dossetor JB, MacLean LD. Histoplasmosis and thrombocytopenic purpura: transmission by renal homotransplantation. Can Med Assoc J. 1965;93(11):587-592. 